\title{
A NEW APPROACH FOR COMPLETE MIXING BY TRANSVERSE AND STREAMWISE FLOW MOTIONS IN MICRO-CHANNELS
}

\author{
MUH-RONG WANG, CHIAU-YI DAI AND YANG-SHENG HUANG \\ Institute of Aeronautics and Astronautics, National Cheng Kung University, \\ No.1, University Road, Tainan City 701, Taiwan \\ wangmr@mail.ncku.edu.tw
}

\begin{abstract}
Mixing control is an important issue in micro-fluid chip applications, such as $\mu$ TAS (Micro-Total Analysis System) or LOC (Lab-on-Chip) because the flow at micro-scale is highly laminar. Several flow control schemes had been developed for complete mixing in the micro-channels in the past decades. However, most of the mixing control schemes are performed by utilizing specific excitation devices, such as electrokinetic, magnetic or pressure drivers. This paper investigates a new control scheme which is composed of a series of flow manipulation by changing the pressure at the two inlets of the micromixer as the external excitation. The fluids from two inlets are introduced to a square mixing chamber, which provides a space where the streamwise and transverse flow motions take place. The results show that the micromixer can be used to produce a large recirculation zone with series of small transverse fringes under external excitations. It is found that this new flow pattern enhances mixing processes at the micro-scale. A complete mixing can be achieved under appropriate flow control with the corresponding design.
\end{abstract}

Keywords: Micromixer; recirculation; excitation; transverse fringes.

\section{Introduction}

Micro-mixers are the important components in micro-fluidic systems. Applications for micro-fluidic system, such as chemical analysis and medical synthesis, usually involve premixing of various reactants before processing. In the last decade, development of micromixer became an important issue because of its benefits, such as ultra-short mixing time, high heat-exchanging efficiency and safety. The flow in the microchannel is laminar and highly symmetric in nature. Hence mixing at microscale is not as facile as it is at large scale. In order to enhance the mixing performance at microscale, varieties of the working principles have been developed in the literature. Active type micromixers apply external energy such as ultrasonic ${ }^{1}$, magnetic ${ }^{2}$ and electrokinetic ${ }^{3}$ to disturb the micro-fluidic flow, On the other hand, the mixing process of the passive type micromixers relies on the diffusion or chaotic advection mechanisms. The most popular mixing scheme is the lamination of fluid. However, the complex 3D structures associated with those micro-mixers exert the difficulties in the fabrication and result in higher pressure drop. As compared to the 3D structure, 2D fabrications are more attractive due to its benefits, such as the simple fabrication process, less material consumption and easy integration with other components. Recently lots of designs are associated with in-plane 
fabrication, such as spiral ${ }^{4}$, bended ${ }^{5}$ and baffled types ${ }^{6}$. Most of them are involved with the concept of Dean Vortex ${ }^{7}$.

The goal of this paper is to investigate the influence of the transverse and stream-wise flow motion on the mixing performance with square mixing chambers. The micromixer is easily fabricated and is highly adaptive to other components due to its handy operation.

\section{Experimental setup}

The micromixers were made by the micro-fabrication process of computerized numerical control (CNC) milling machines and its dimensions are shown in Fig. 1. The micromixer is designed with a mixing chamber in the down-stream of the Y-shape channel. The depth of the micro-channels is $400 \mu \mathrm{m}$ in all cases. The experimental setup is also illustrated in Fig. 1. It consists of an image acquisition system and an external excitation device. Reynolds number, $\mathrm{Re}=\mathrm{VD} / \mathrm{v}$, was controlled by the pulling rate of the syringe pump located in the up-stream of the micromixer. External excitation was provided by two home-made piezo-electric vibrators. Images were acquired by the high speed cameraRedlake-X4.
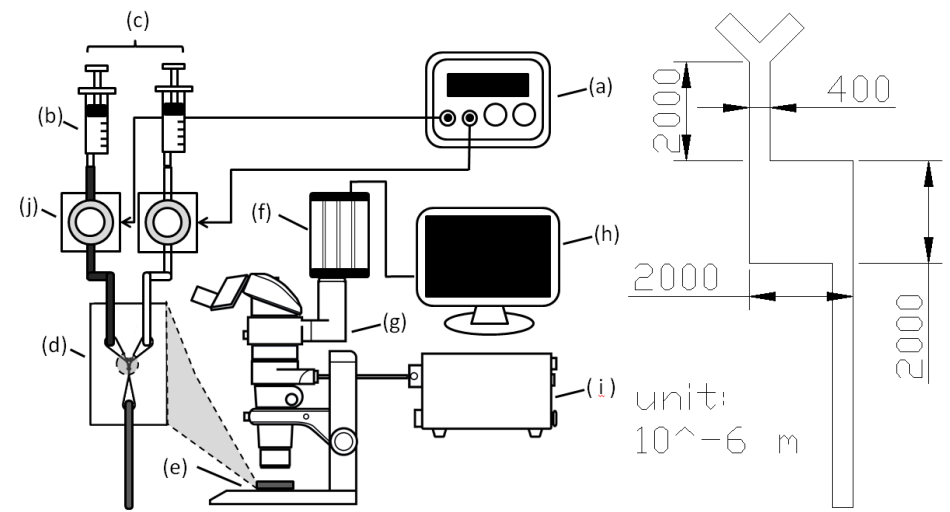

Fig. 1. (a) function generator (b) two ports syringe pump-KDS200 (c) inlets (d) micromixer (e) micromixer holder (f) redlake-X4 high speed camera (g) Nikon-SMZ800 microscope (h) computer (i) 150w lamp (j) pizeoelectric vibrator $(\mathrm{k})$ dimensions of the micro-mixer.

\section{Flow Visualization}

Flow visualization was performed by the high speed camera with a fixed sampling rate of 487 frames per second. Figure 2 illustrates the mixing processes of the micromixer under $\mathrm{Re}=1$ and excitation frequency of $25 \mathrm{~Hz}$. As shown in Fig. 2a, Mixing in the micromixer without external excitation is essentially relied on the molecular diffusion. Fig. $2 b$ shows a vortex pattern was generated under external excitation. The vortex pattern was then transported to the downstream of the mixing chamber, as shown in Figs.2d and 2e. The vortex shape mixing pattern was stretched and laminated in this step. The mixing process of this micro-mixer under external excitation can be summarized as follows: (1) the vortex shape mixing under external excitation, the piezo-electric vibrators result in the 
suction force in the upper stream and the down-stream fluids were thrust into the mixing chamber at the same time. Thus the mixing chamber provides a free space to form a vortex-shaped mixing pattern during this process. The existence of the vortex-shape mixing pattern also means that the transverse flow motion takes place in the micro-mixer. (2) The piezo-electric vibrator is then create a positive pressure in the upper stream, thus the fluids in the mixing chamber were pushed to the micro-channel in the down-stream.

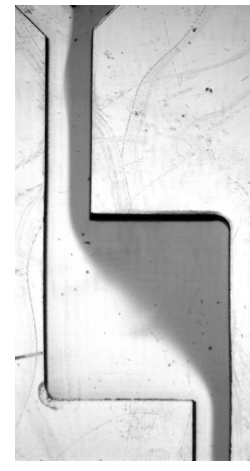

(a) 0 cycle

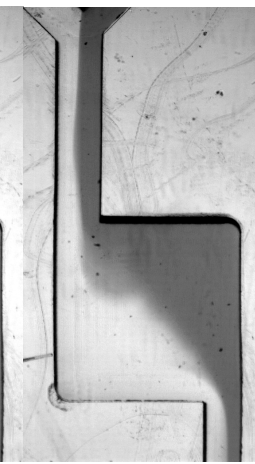

(b) $1 / 4$ cycle

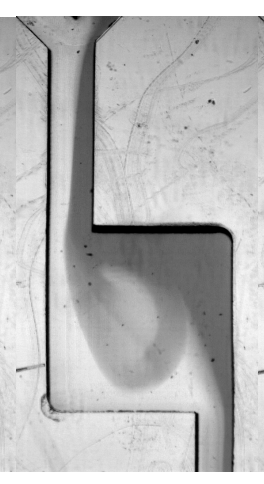

(c) $1 / 2$ cycle

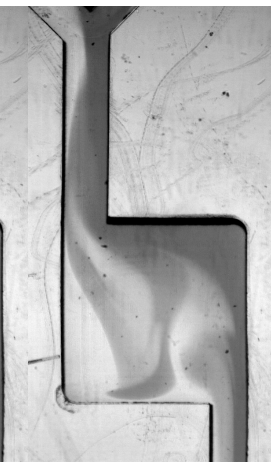

(d) 3/4 cycle

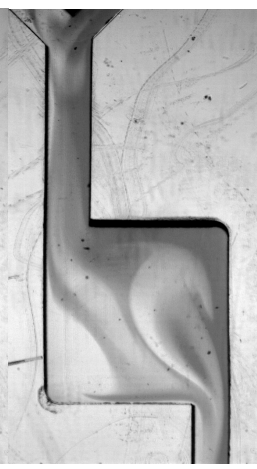

(e) 1 cycle

Fig. 2. Mixing process in the mixing chamber within the first excitation cycle $(f=25 \mathrm{~Hz})$.

The flow field was stretched in this process due to the area change in the flow direction. Flow lamination takes place in the mixing layers. Fig. 3 shows the mixing pattern in different cycles. Comparing Fig.3a with Fig.3d, the boundary between laminated fluids becomes blurred. Figs. $3 \mathrm{e}$ and $3 \mathrm{f}$ also reveal that the mixing performance stops increasing at the $5^{\text {th }}$ cycle because of the similarity of flow pattern in the micromixer. As can be seen in Fig. 4, the flow patterns and mixing performance were strongly influenced by the external excitation frequency. Under lower excitation frequency, such as $\mathrm{f}=12.5 \mathrm{~Hz}$, the size and the number of laminated mixing layer of the vortex-shape flow is much less than that under higher excitation frequency, such as $f=20$ and $\mathrm{f}=25 \mathrm{~Hz}$. Hence more folding and stretching processes in the micro-mixer take place under higher excitation frequency.

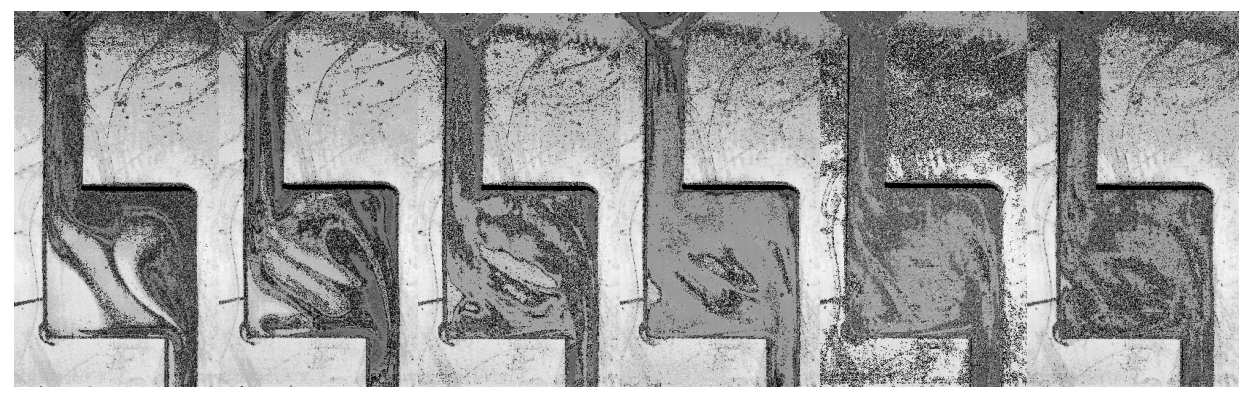
(a) $1^{\text {st }}$ cycle
(b) $2^{\text {nd }}$ cycle
(c) $3^{\text {rd }}$ cycle
(d) $4^{\text {th }}$ cycle
(e) $5^{\text {th }}$ cycle
(f) $6^{\text {th }}$ cycle

Fig. 3. Mixing patterns in different mixing cycles under excitation frequency of $25 \mathrm{~Hz}$. 


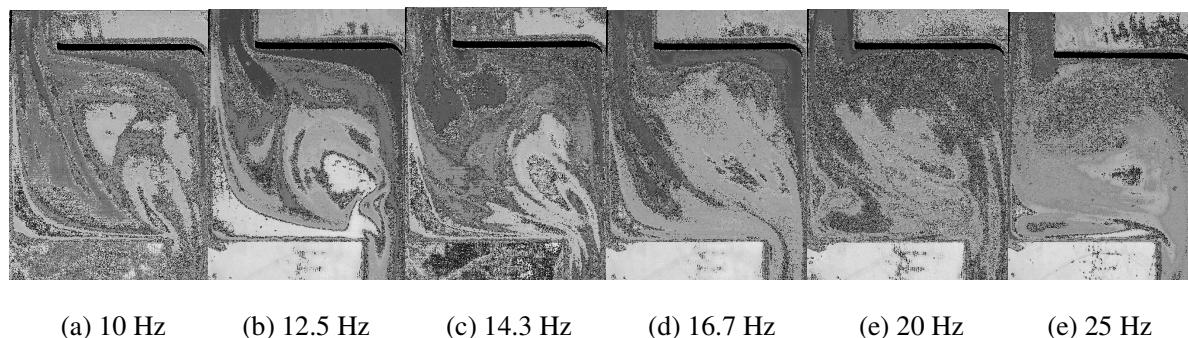

Fig. 4. Comparison of mixing patterns under varied excitation frequencies after three mixing cycles.

\section{Mixing Efficiency}

The evaluation of mixing efficiency, which is based on the theory of Danckwerts' intensity of segregation ${ }^{8}$, can be refined as $\eta_{\text {mixing }}=1-\left[\Sigma\left(C_{x}-C_{r e f}\right) /\left(C_{m a x}-C_{r e f}\right)\right] / n$. In this paper, mixing efficiency was evaluated at the cross section A-A, which is at the exit of the mixing chamber (see Fig. 4b). It seems that the mixing process under very low Reynolds number was totally relied on molecular diffusion without external excitation. As can be seen from Fig. 5, the mixing efficiency as low as $13.1 \%$ is found under $\mathrm{Re}=1$ without external excitation. Under excitation frequency of $25 \mathrm{~Hz}$, the mixing efficiency was dramatically raised to $49.4 \%$ in the first cycle and the mixing efficiency was increased to $95.1 \%$ at the $12^{\text {th }}$ cycle. The mixing efficiency became stabilized after the $16^{\text {th }}$ cycle with an average mixing efficiency of $90 \%$. The mixing efficiency higher than $90 \%$ is normally considered as complete mixing in the micromixer.

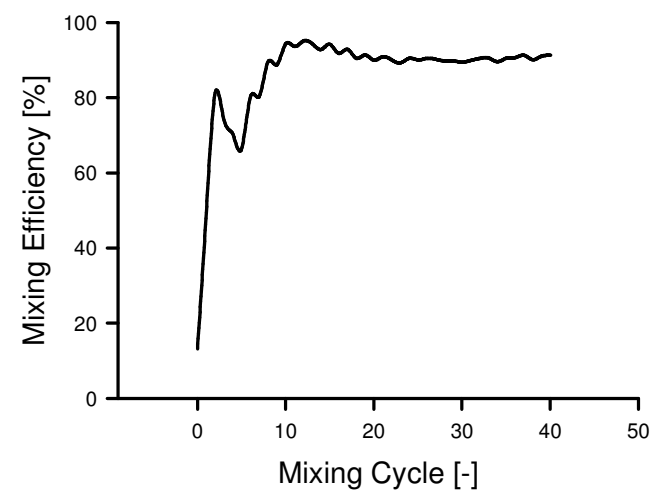

Fig. 5. Dependence of the mixing efficiency on the mixing cycle under $\mathrm{f}=25 \mathrm{~Hz}$.

\section{Conclusions}

The mixing phenomena in the micromixer with square mixing chamber were described under external excitation. Comparing with other types of the micro-mixers, our design is simple and more effective. It can achieve the complete mixing within ultra short mixing length. The mixing efficiency higher than $90 \%$ at $\mathrm{Re}=1$ is achieved within one set of the 
square mixing chamber. The can be concluded that the mixing processes have been significantly enhanced with this novel design of the micromixer.

\section{References}

1. W. K. Tseng, J.L. Lin et al., J. Micromech. Microeng. 16, 539 (2006).

2. D. W. Oh, J. S. Jin et al., J. Micromech. Microeng. 17, 2077 (2007).

3. L. M. Fu, C.H. Tai et al., J. Phys.: Conf. Series, 34, 45 (2006).

4. Y. H. Hu, M. Chang et al., J. Phys.: Conf. Series, 48, 531 (2006).

5. J. T. Yang, K.-W. Lin et al., J. Micromech. Microeng. 16, 2439 (2006).

6. A. A. S. Bhagat, E. T. K. Peterson et al., Microfl. BioMEMS, Med. Microsys. 61120J-61110.

7. W. R. Dean, Proc. Roy. Soc. London. 121, 402 (1928).

8. P. V. Danckwerts, App. Sci. Res. A3, 279 (1952). 